\title{
Towards Sustainable Agriculture: Current Perspectives for Future Sustainability
}

\author{
W.C.P. Egodawatta
}

Editor,

Sri Lankan journal of Agriculture and Ecosystems, Faculty of

Agriculture,

Rajarata University of Sri Lanka

Correspondence:

*egowcp@agri.rjt.ac.lk

iD https://orcid.org/0000-0003-3934-6351

DOI: http://doi.org/10.4038/sljae.v2i2.35
Sustainable agriculture has multiple definitions and multiple approaches leading to an enormous diversity; thus, it may be difficult to synthesise meaningful comparisons between these approaches. These approaches have certain goals in common, mainly designed and aimed for sustainability, which includes multidimensional environmental aspects as well as regional and local socio-economic considerations. Despite the nature of approaches, all target the sustainable agriculture in various degrees, accounting a better state of the environment, safe and healthy food, and a good life.

These approaches, principally, lead to a multidimensional improvement of an agroecosystem contrast to the conventional agriculture. The conventional agriculture, in longer term, resulted negative impacts on the environment and create a struggle to be competitive in farming context. Generally, public believes healthy food as an outcome and benefit of an environmentally friendly farming approach. Hence, approaches have been developed to ensure more ethical way of farming, even now the focus has been shifted towards animal welfare as well. The greatest strength of sustainable approach is the accessibility to smallholders. In contrary, sustainable farming approaches have been criticised in many developing nations due to low expected outcomes and limited profitability. Further, in many of such programmes, the scalability of application has also been widely debated. Scientists have also accepted such criticisms, and among the general farming community, these confusions and misunderstandings has led to suboptimal implementation, regionally, globally.

This is an open-access article distributed under the terms of the Creative Commons Attribution 4.0 International License, which permits unrestricted use, distribution and reproduction in any medium provided the original author and source are credited. 
Implementing a sustainable approach shall have definitions and understanding its own by adhering to the core goal and objectives in a unique way. Especially, in certain approaches the economic sustainability has been prioritised over environmental, hence the perceived benefits may not feasible from farming conditions. In these instances, it would be problematic or rather false to claim that the implementation of an approach highlights as sustainable agriculture, when perceived environmental goals are not met.

Many of the more holistic approaches of sustainable agriculture aim to generally address common issues through various practices. Restoring soil health, reducing resource consumption, minimising vulnerability to pests in environmental-friendly ways, reducing pollution and GHG emissions, and protecting and restoring biodiversity are at upfront as focal principles. In general, sustainable agricultural practices are fundamentally in terms of environmental-friendly practices that are useful in maintaining the unity between systems, and in broad sense of restoring the biodiversity as well.

Basically, most of scientifically proven systems were very common in historical perspectives of farming prior to the establishment of scientific or technology dependent agriculture, widely adopted such approaches are,

- Crop rotation (nitrogen fixing crops and cereals)

- The inclusion of cover and companion crops

- Mixed crop and intercropping or various spatial arrangements

- Restricted or no use of synthetic pesticide and mineral fertilisers

- Zero or minimum tillage

\section{- Free ranged livestock}

Further, many alternatives are incorporated to technological agriculture even in current context, in different degree, in order to fulfil objectives of sustainability. The methods such as crop diversification, mixed crop and integrated animal farming, agroforestry, manure substitutions and sitespecific nutrient management, recycling of biomass and residues and the inclusion elements such as hedgerows and strips are common forms in tropical to temperate zones. These practices are solely or combinedly appear in many systems, while for certain the number of methods might be longer for some of the approaches. Hence, in many instances the presence of one or more practices mentioned are considered as a sustainable agricultural approach.

\section{Challenges Foreseen:}

Despite the method or methods uses, a critical understanding on specific challenges per each approach, and their potential or their emphasis on all three pillars (i.e., environmental, social, and economic) of sustainability needs to be assessed. In many instances, the fundamentals of application are similar and beyond and that the challenges have also been common. These common challenges have to do with costs, profitability, productivity, scalability, acceptance, knowledge, and in many cases, environmental sustainability.

Many of these challenges are interlinked, i.e. costs are inevitably linked to profitability, scalability, and so on. Although, it leads to a greater complexity in understanding, addressing one challenge can end up addressing many; which are interlinked. Widely criticised phenomena of sustainable agricultural 
approaches are the associated costs, particularly in terms of labour and knowledge.

Hence, challenge inseparably linked with costs is not only profitability, but also the productivity. Yield gaps are the most tested phenomena for many approaches, affecting their economic sustainability. Undeniably, substitution of mineral fertilisers and synthetic pesticides has an impact on expected yields of many systems. In general perception, the agricultural industrialization is more profitable, thus heavily driven over time by economics and technology.

Conversely, agroecology, include promotion of the production of locally adapted products in a greater variety rather than in large volumes of one or a few standardised species and varieties. Despite the critiques of productivity, scalability, and profitability, premiums produce outweigh the costs of the approach; although, it could be argued that premiums may only be placed in a smaller niche market.

When considering the challenges, ultimately these approaches can safeguard the sustainable global economy. Hence, the potential costs and/or a reduction in profitability might be alleviated with public or private interventions in such transition towards sustainable land use and resource use. Providing a support can save the adopters from early negatives of societal and economic pressure. In many countries, most conventional agricultural practices are heavily subsidised by governments, without which their profitability and sustainability might also be compromised.

A critical factor behind a common challenge related to knowledge is the lack of indicators, especially important in convincing. The lack of evidence of the benefits of these approaches, unable to convince neither farmers nor, consumers, nor policy. Most instances, the environmental impacts or benefits of certain solutions are not easily measurable, and thus difficult to ascertain. As every approach has its own angle, a particular approach might have challenges giving all crucial environmental aspects the same level of consideration, i.e. soil, water, biodiversity, and the climate being the major aspects. While, in some cases, this might mean simply less of a focus on a given aspect over another, in other cases a sole focus on one aspect may lead to negative outcomes for another. The environmental sustainability would need to consider and strive for the better state of all key aspects in a balanced, integrated manner.

\section{Practicing Sustainable Agriculture:}

When choosing a particular way to produce foods, in terms of suitability and environmental sustainability, two major questions are to be concerned.

1. Whether the approach is suited with the landscape and environmental conditions in which it would be practiced (spatial consideration)? As an example, an area at the vicinity of a protected wildlife or nature sanctuary, and agroecological approach, while blending the use of buffer zones and existing farmland for food production and conservation simultaneously (sustainable/ ecological intensification).

2. Will the use of sustainable agricultural approach make the environment better than before (temporal consideration)? It should not only be the environment, the livelihood or the socio-economic context should be uplifted from existing, as the 
intervention should be a way forward than a destructive force.

As an example, conversion of sustainably thriving a perennial based production system with a high demanding spice crop, despite integrating to the existing system can be a serious threat to the associated biodiversity, and further cause an exhaustive pressure on soil and water reserves of the system. Such conversion may attract farmers in short-run due to early profits, however altogether the costs can be higher with an assessment of its impact to the environment.

The key elemental questions must be addressed not only by the farmer himself, a broader participation of stakeholders along the food and values chain is necessary in useful implementation. The context of social and economic sustainability of sustainable agricultural approaches is more likely to be rational, when there is an emphasis on livelihoods farthest down the food value chain, rather than on profit, currently captured higher up the chain.

\section{Future Perspectives}

Many approaches of sustainable agriculture may produce healthier, more nutritious food, and ethical concerns such as raising animal welfare. The lower costs of highly processed and junk foods always carry a hidden societal cost and do include a high environmental footprint. Thus, the foods from sustainable approaches may require a better regulation along the food value chain to ensure perceived benefits. Hence, sustainable food production not just a localized effort of a small group, and it will bring a global partnership such as understanding of global consumption patterns, key trade attributes, and ecological footprint of similar systems around the globe.

Local, regional, and national activities and policies will continue to influence the global picture, and vice versa. In this respect, the COVID-19 crisis has shown some interesting examples of how global supply chains may have given way to more local production and consumption chains in food systems.

Globally, a common vision will be the stepping stone for sustainable agricultural approaches. Such initiative may encourage many transitional changes to current agroecosystems to a shape in which it would in the future. This is a fundamental question, considering the main societal goal for agriculture to produce food, the necessary use of land for this purpose, and the negative impacts that this activity can have on the environment.

A comprehensive review of global sustainable agriculture systems in, diverse environments, contrasting societies, and distinct economies have proven that an immeasurably diverse approaches are possible, with many commonalities. The implementation depends very much on local contexts and specific priorities of society and economy.

The test is to formulate better policies, create the atmosphere for enabling a dialogue of stakeholders of sustainable agriculture transitions in defining priorities according to local contexts, assisting the adopters in a socially desired pathway with economic success. Considering the production of healthy foods at reasonable values, while protecting environment is highly vital for our existence as mankind, thus, addressing these challenges is the most important step to leap for a better future. 